\title{
The role of telomere shortening in the development and progression of COPD
}

\author{
Hoda Mojazi Amiri MD, Jason P. Cooper MD, PhD, Kenneth Nugent MD
}

\begin{abstract}
The prevalence and incidence of COPD increase with age in all populations studied. These associations suggest that normal aging could contribute to the pathogenesis of COPD. Telomeres are DNA structures at the end of chromosomes which protect DNA from degradation and unneeded recombination events. Telomere length decreases with age. At a critical length cells undergo senescence which results in changes in cell morphology, gene activity, and cytokine production. Senescent cells eventually undergo programmed cell death (apoptosis) and drop out of tissue structures. Telomeres from lung tissue and circulating leukocytes are shorter in patients with COPD. Lung biopsy specimens indicate that patients with COPD have increased numbers of apoptotic epithelial and endothelial cells. This information suggests that normal aging or accelerated aging or senescence induced by oxidant injury and/or inflammation contributes to the development of COPD. Longitudinal studies suggest that patients with shorter telomere have increased all cause and cancer mortality. Therefore, telomere attrition is associated with important consequences in these patients. In addition, animal studies suggest that patients with shorter telomeres are at increased risk of developing COPD. These observations raise the possibility that drugs designed to increase telomere length or to prevent telomere attrition might slow the progression of COPD. However, very careful studies with animal models of COPD and of malignancy are needed to determine benefits and to make certain that this genetic manipulation does not increase the risk of malignancy.
\end{abstract}

Key words: normal aging, accelerated aging, telomeres, telomerase, cellular senescence, COPD

\section{INTRODUCTION}

Chronic obstructive pulmonary disease (COPD) is a leading cause of morbidity and mortality worldwide. In the recently published Global Burden of Disease Study 2010, COPD rose from the fourth leading cause of death in 1990 to the third leading cause in 2010 and was the ninth leading cause of years of life lost ${ }^{1}$. Across all age groups, COPD was

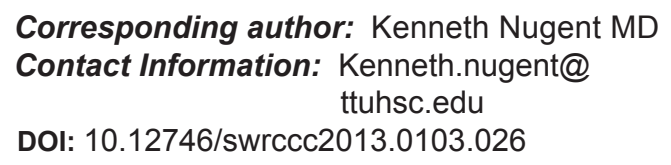

estimated to cause 2.9 million deaths worldwide in 2010 , and prevalence studies suggest that $10 \%$ of all adults aged 40 and older have stage II (moderate) or higher airflow obstruction based on the Global Initiative for Chronic Obstructive Lung Disease (GOLD) guidelines $^{1,2}$. By 2030, COPD is projected to be the direct underlying cause of $7.8 \%$ of all deaths and will represent $27 \%$ of deaths related to smoking ${ }^{3}$. The essential clinical characteristic of COPD is irreversible expiratory airflow limitation measured by spirometry. These patients usually have chronic bronchitis, chronic bronchiolitis, and/or emphysema 
present on pathological studies. The pathogenesis involves oxidative stress which causes chronic low grade inflammation in the lung parenchyma, protease-antiprotease imbalance with elastin degradation, impaired tissue repair, and systemic inflammation. These processes are, of course, complex and likely vary from patient to patient. Studies on pathogenesis need to explain why COPD develops in a minority of cigarette smokers, why COPD can progress in some ex-smokers, and why COPD can develop in non-smokers. Both cigarette smoke and other oxidative stress promote cellular senescence (a term describing aging at the cellular level), and accelerated or abnormal aging of the lung tissue could contribute to the development of COPD in some patients ${ }^{4,5}$. In this review we will discuss the relationship between age and the prevalence and incidence of COPD, studies on telomere length in patients with COPD, and the relationship between telomere length and cellular senescence and programmed cell death.

\section{AgINg AND COPD}

There is a definite association between COPD and aging, and patients with COPD often present for clinical care in their 60s and 70s. Van Durme and coworkers reported a prospective population based cohort study of subjects older than 55 years living in a suburb of Rotterdam ${ }^{6}$. This study included 7983 participants with a median follow-up of 11 years. The baseline prevalence of COPD was $11.6 \%$. There was a progressive increase in prevalence until age $79(16 \%)$. The incidence during follow-up was 9.2 cases per 1000 person-years. The highest incidence occurred in study participants ages 75-79 at study entry (12.8 cases per 1000 person-years). The incidence was 4.2 cases per 1000 person-years in never smoking men and 3.8 cases per 1000 person-years in never smoking women. The incidence in never smokers was highest in subjects ages 70-79 at study entry. Lamprecht et al reported a 14 country population based study using spirometry and respiratory questionnaires to determine the prevalence of COPD based on the GOLD criteria ${ }^{7}$. COPD occurred in all countries, was more frequent in current smokers and men, and increased with age. Twenty-three percent of the participants with GOLD stage II + COPD were never smokers; $12.2 \%$ of never smokers had GOLD stage I+ COPD. Risk factors for COPD in never smokers included age, work (>10 years) in a high risk occupation, a history of asthma, and a low BMI $(<20 \mathrm{~kg} / \mathrm{m} 2)$. These studies and others demonstrate that COPD is, at least in part, related to aging ${ }^{8,9}$. This could reflect cumulative particle exposure and the slow accumulation of lung injury over years. It could also reflect an interaction between aging and the pathogenetic processes causing lung injury in smokers. Lung function declines in healthy individuals with age. Pathology studies demonstrate that alveolar dimensions increase, the gas exchange surface area decreases, and elastic tissue supporting peripheral airways decreases. These morphological changes cause predictable changes in lung function with aging. The total lung capacity is stable, but the forced vital capacity falls, and the residual volume increases 10. Since there is no alveolar wall destruction and no bronchial inflammation, age related changes do not reproduce the usual pathological changes seen in COPD. Hence, COPD in non-smokers is not due just to accelerated aging. But the normal processes associated with aging could contribute to the development of lung disease in both smokers and nonsmokers. Telomere shortening occurs in normal and accelerated aging and in smokers and patients with COPD. It provides a biological marker for aging and has a critical role in cellular senescence and cell death. The latter two processes could promote disease progression.

\section{TELOMERE SHORTENING MECHANISMS AND}

\section{LENGTH DETERMINANTS}

Telomere shortening and replicative senescence may contribute to diverse diseases, including cancers, cardiovascular diseases, neurodegenerative diseases, and COPD. Telomeres are DNA structures and associated proteins at the ends of chromosomes 11. They are made of non-coding double-stranded repeats of 5'-TTAGGG-3' DNA sequences, which are $9-15,000$ bases pairs in humans. They help stabilize DNA and prevent its degradation and gene-to-gene recombination. Telomeres are associated with a group of proteins, called the shelterin complex which includes TRF1, TRF2, POT1, TIN2, TPP1, and Rap 1. This complex recognizes TTAGGG repeats and 
prevents the DNA repair pathway from recognizing them as DNA breaks. Telomere repeats shorten by 30 to 200 base pairs with each cell division in mature somatic cells due to end-replication problems (the location of the DNA polymerase prevents the synthesis of terminal DNA sequences). When telomeres reach a critical length, their ability to protect the DNA decreases, and cell cycle arrest occurs. These cells enter senescence; they have a change in morphology and a change in gene expression. P53 and p16 pathways are activated, and this leads to programmed cell death or apoptosis. Cellular senescence occurs naturally and represents the end result of the replicative capacity of a cell. It can also occur after oxidative injury or as a consequence of inflammation. Therefore, telomere length is an indicator of biological age ${ }^{12}$.

The enzyme telomerase prevents telomere shortening. This enzyme is a ribonucleoprotein whose RNA can provide a template for elongation of telomere ${ }^{13,14}$. The core enzyme includes a reverse transcriptase and a RNA sequence containing 451 nucleotides. It is active in germ cells and stem cells (and cancer cells) but is less active or inactive in fully differentiated cells. The alternative length pathway can also maintain telomere length, and this pathway involves homologous recombination mediated DNA copying of the telomeric DNA template ${ }^{15}$.

There is high degree of correlation in the telomere length of white blood cells, umbilical cord cells, and skin of a newborn ${ }^{16}$. In adults, there is high interindividual variability in telomere length, and telomere lengths in different tissues in individual patients are variable ${ }^{17}$. These differences in length among individuals are controlled, in part, by heredity and likely involve multiple genes ${ }^{18,19}$. However, Huda reported that the length of the telomeres did not correlate well in monozygotic twins. Rather, persons who shared environmental factors had higher correlations in telomere length ${ }^{20}$. Therefore, environmental factors also influence telomere length. For example, Morla et al found that cigarette smoking has a doseresponse relationship with telomere shortening and suggested that systemic inflammation and oxidative stress due to smoking could explain this effect ${ }^{21}$. Oxidative stress causes double-strand breaks in telomeres possibly because of the high guanine content ${ }^{22}$. Guanine is more sensitive to oxidative stress than other bases because of its low oxidation potential ${ }^{23}$ and is more likely to be oxidized when in sequence of GG or GGG compared to a single G ${ }^{24,25}$.

Women aged $20-80$ have longer telomeres than men in the same age group; there are no significant differences in telomere lengths of the newborns with regard to gender ${ }^{26,27}$. These gender differences may be explained by the effect of estrogen on telomerase activity, and Misiti et al have demonstrated that estrogen upregulates the transcription of hTERT, the catalytic subunit of human telomerase ${ }^{28}$. These differences associated with gender may help explain the differences in the incidence of COPD in men and women, but the complexity of interactions among gender, telomere length, and disease progression is beyond the scope of this review.

\section{MEASURING TELOMERE LENGTH}

Several assays can measure telomere length, including Southern blot, fluorescence in-situ hybridization (FISH), and quantitative-polymerase chain reaction (q-PCR) based assays ${ }^{29}, 30$. While Southern blotting, which infers telomere length from the optical density of telomere-region restriction fragments, remains the gold standard, the time-consuming nature and low throughput of this procedure combined with inaccuracies from the inclusion of subtelomeric regions have led investigators to evaluate other methods for measurement. Quantitative-FISH (q-FISH), also a complex and labor-intensive technique, offers more precise quantitative measurement based on calculation of the number of telomeric repeats from fluorescence intensity that varies with the number of telomere-specific nucleic acid probes that are attached. However, because only the telomeres of chromosomes in metaphase spreads are measured, senescent cells are not included, and this may bias the results toward increased lengths. The combination of flow cytometry with q-FISH (flow q-FISH) shortens assay time and reduces bias by measuring telomere length of cells in various phases of the cell cycle. Flow q-FISH can also include control cells as a telomere length standard, and this al- 
lows comparison of telomere length in different cell/ tissue samples with high precision. Finally, the use of qPCR to determine telomere length has become increasingly popular since the original description by Cawthorn in 2002 29, 31. O'Callaghan improved the measurement of telomere length through quantitative real-time PCR (qRT-PCR) which utilizes 84mer oligonucleotides containing repeats of TTAGGG in known quantities to generate a standard curve from which absolute telomere length from a cell/tissue sample can then be calculated ${ }^{32}$. Multiple investigators have used qRT-PCR in recent studies of telomere length determination in peripheral leukocytes of patients with COPD ${ }^{33-36}$. Given its accuracy combined with the increasing availability of automated systems that can accommodate 384-well plates for high throughput screening (e.g., Applied Biosystems $7900 \mathrm{HT}$ ), qRT-PCR will likely be the preferred assay for telomere length measurement in future studies.

\section{Telomere LeNGTH AND COPD}

Several studies have reported that telomere length is shorter in patients with COPD than in healthy controls ${ }^{36-38}$. Rode et al ${ }^{35}$ tested 46,396 individuals, including 6,770 with COPD, from a general Danish population. They found that telomere length decreased with age, participants with low FEV1/FVC ratios had shorter telomeres, and participants with COPD had shorter telomeres. Subjects with shorter telomeres were 2.06 fold more likely to develop COPD; after adjustment for confounding factors, this risk decreased to 1.15 fold. Mui et al studied 283 patients in a COPD drug trial ${ }^{38}$. Telomere length was shorter in older patients and in patients with lower FEV1/FVC ratios. In addition patients with higher circulating levels of surfactant protein $D$ (an inflammatory marker from the lung) had shorter telomeres. Savale et al compared telomeres length in 136 COPD patients, in 113 age and sex-matched controls, and in 42 nonsmokers without COPD ${ }^{36}$. Patients with COPD had shorter telomeres independent of age, sex and pack-year smoking history, and COPD patients had increased levels of circulating interleukin 6 (a marker of inflammation). The decreased length in COPD occurred in all age groups in this study. Houben et al studied 102 patients with COPD and 19 age matched controls and found that patients had shorter telomeres ${ }^{37}$. These patients also had lower circulating levels of superoxide dismutase which is an antioxidant. Low levels of this enzyme might contribute to increased oxidant related injury in the lung in COPD patients. Morla et al reported that smokers had decreased telomere lengths and that there was a dose-effect relationship between telomere length and cumulative long-life exposure to smoking ${ }^{21}$. This study did not find shorter telomeres in smokers with COPD, but the number of subjects was small. Cigarette smoking did not appear to influence telomere length in studies reported by Savale and Houben, but these patients all had COPD and the inflammatory processes associated with active disease may have had a greater effect on telomere length than cigarette smoke alone. The relationship between COPD severity (based on the pulmonary function tests) and telomere length has been inconsistent in the recent studies, and Houben and Savale did not find a relationship between lung function parameters and telomere length ${ }^{36,37}$. However, Rode did find that participants in the Danish study with lower FEV1/ FVC ratios (more obstruction) had shorter telomeres.

Lee et al reported that patients with shorter telomeres are at increased risk for all cause and cancerrelated mortality ${ }^{34}$. These investigators measured the telomere length in 4271 patients with mild to moderate COPD and analyzed survival over the next 7.5 years. Patients in the three quartiles with the shortest telomeres had a higher risk for mortality from cancer with a hazard ratio of 1.48 and a higher risk for mortality from all causes with a hazard ratio of 1.29 when compared to the quartile with the longest telomeres. Smoking did not appear to affect the telomere length in the patients with definite COPD. Therefore, telomere length may be an indicator of susceptibility to cancer and mortality in COPD patients.

In summary, patients with COPD have shorter telomeres in circulating leukocytes and lung tissue (discussed below) and short telomeres are associated with an increased all cause and cancer mortality in these patients. 


\section{TELOMERES AND THE PATHOGENESIS OF COPD}

It is unknown whether telomere shortening is a cause or a consequence of COPD. Oxidative stress and inflammation are important factors in telomere attrition in these patients. In Savale's study, the level of IL-6 was significantly higher in COPD patients with shorter telomeres ${ }^{36}$. In Houben's study, the activity of superoxide dismutase was significantly lower in patients with shorter telomeres ${ }^{37}$. These results suggest that shorter telomeres in COPD patients are the result of increased oxidative stress and inflammation in these patients, rather than shorter telomeres having a causal effect in the development of COPD. However, animal studies suggest that telomere shortening contributes to the development of COPD. Alder studied mice with null telomerase and found that mice with shorter telomeres were more likely to develop emphysema when exposed to chronic cigarette smoke than control mice ${ }^{39}$. Lee studied four generations of mice null for telomerase to measure the effect of telomere shortening on lung tissue and found that the mice with the shortest telomeres developed alveolar wall thinning and increased alveolar size ${ }^{40}$. These studies suggest that telomere shortening could make individuals more susceptible to the development of emphysema and lowers the threshold for damage induced by cigarettes. This process involves cellular senescence and programmed cell death.

Normal aging causes cell loss in alveolar-ca pillary units; inflammation in the lung also leads to cell loss. Alveolar cells replicate to replace these cells but eventually cannot keep up with replacement because of replicative senescence. Then, lost alveolar cells are not replaced, and alveoli increase in size and the surface area decreases. These events create emphysematous changes in lung tissue. Cellular senescence develops during normal aging, during accelerated or abnormal aging, and as a consequence of oxidative injury. Reactive oxygen species can damage cells by directly damaging DNA and shortening telomeres, or by activating transcription factors, such as NF$\mathrm{kb}$ which leads to a pro-inflammatory state. Senescent cells release cytokines which stimulate inflammation and perhaps perpetuate it in the absence of ongoing toxic exposures. Studies with lung tissue specimens demonstrate that patients with COPD have increased apoptosis in both endothelial and epithelial cells, and the presence of these cells supports the hypothesis that normal aging and abnormal aging could contribute to the development of emphysema through limitations of lung repair following oxidative injury ${ }^{5,41}$.

\section{TELOMERE AS PHARMACOLOGIC TARGETS}

Since telomere shortening is a major determinant for the onset of cellular senescence, telomerase has been considered an attractive target to lengthen telomeres and potentially delay or prevent disease related to aging, including COPD. Cellular senescence was accelerated in both alveolar type 2 and endothelial cells of patients with emphysema, as compared to those in asymptomatic smokers and non-smokers ${ }^{5}$. Additionally, studies in telomerase-null mice exposed to cigarette smoke showed that shortened telomeres were sufficient to cause emphysematous air space enlargement regardless of the extent of the inflammatory response ${ }^{39}$.

Currently, the only developed pharmacologic activator of telomerase is cycloastragenol (TAT2, CAS Registry no. 84605-18-5), a small molecule sapogenin developed by Geron Corporation and TA Therapeutics, Ltd. Only in vitro studies have been reported, but TAT2 has been shown by two groups of investigators to increase telomerase 
activity levels, retard telomere shortening, increase proliferative capacity, and enhance functional secretion of cytokines in human CD4 and CD8 T lymphocytes ${ }^{42,43}$. Other phytochemicals, such as resveratrol, genistein, sulforaphane, and silibinine, are also direct or indirect activators of telomerase, and resveratrol has been shown to do so in vitro via upregulation of the NAD-dependent deacetylase SIRT1 in human mammary epithelia and endothelia progenitor cells ${ }^{44-46}$.

In their classic treatise on the biological hallmarks of cancer, Hanahan and Weinberg cite the re-activation of telomerase to protect chromosomal telomeres in cancer cells as being centrally involved in the acquisition of the capability for unlimited proliferation ${ }^{47,48}$. The connection between inappropriate telomerase activity and tumorigenesis is an obvious concern in the use of exogenous telomerase activators, particularly in current or former smokers who are at greater risk for the development of multiple types of cancer and whose cells may already have tumorigenic genetic alterations from the oxidant/inflammatory effects and/or the carcinogens in cigarette smoke. Fauce et al noted that the TAT2-induced telomerase activation in $\mathrm{T}$ lymphocytes was short term in that telomerase activity returned back to baseline within a few days of stopping treatment with TAT2 ${ }^{42}$. However, whether these results extrapolate to other cell types in vitro or to in vivo models of COPD is unknown. More in vitro and in vivo testing is needed to assess both the potential efficacy and consequences of the pharmacologic modulation of telomerase, particularly in animal models of premalignancy to estimate any risk of tumorigenesis.

\section{Conclusion}

COPD predominantly occurs in older individuals who have smoked or have been exposed to environmental toxins. Both normal and accelerated aging and cellular senescence could contribute to the development of this disease. Somatic cells enter senescence pathways when their telomeres reach a critical length; these senescent cells can no longer support tissue repair and release cytokines which could promote ongoing inflammation in lung tissue. These cells eventually undergo programmed cell death and drop out of lung tissue. Patients with COPD have shorter telomeres in circulating leukocytes and in lung parenchymal cells. These observations suggest that the oxidant stress in COPD promotes cellular senescence or that accelerated aging contributes to the pathogenesis of COPD. Animals with decreased telomerase activity are more susceptible to cigarette smoke and develop more emphysema than control animals. Therefore, drugs that inhibit telomere shortening or stimulate telomerase activity could alter the progression of lung disease in patients with COPD. However, any studies using drugs with these effects would require careful attention to the development of cancer in patients already at risk for cancer in the lung and other tissues.

\section{Key Points}

1. Normal and abnormal aging may contribute to the pathogenesis of COPD, especially in ex-smokers and nonsmokers.

2. The length of telomeres decreases during each somatic cell cycle, and at a critical telomere length cells enter replicative senescence and then undergo apoptosis. 3. Lung cells and leukocytes in patientswith COPD have shorter telomeres. This is an expected consequence of aging and may also develop as a consequence of oxidant injury from cigarette smoke.

4. The loss of cells in the alveolar region from senescence and cell death reduces the number of cells in epithelium and endothelium and 
reduces normal cell replication and tissue repair. In addition, senescent cells release cytokines which could stimulate inflammation.

5. More studies on aging and its relationship to COPD may clarify the pathogenesis of COPD and identify alternative approaches to prevention and/or treatment.

\begin{abstract}
Author Affiliation: Hoda Mojazi Amiri is a research assistant in the Department of Internal Medicine. Jason P Copper completed his medical education at TTUHSC School of Medicine in May 2013. Kenneth Nugent is a pulmonary physician in the Department of Internal Medicine.
\end{abstract}

Received: 3/1/2013

Accepted: 5/28/2013

Reviewers: Vijay Tonk, $\mathrm{PhD}$

Published electronically: 8/ /2013

Conflict of Interest Disclosures: None

\section{REFERENCES}

1. Lozano R, Naghavi M, Foreman K, Lim S, Shibuya K, Aboyans V, et al. Global and regional mortality from 235 causes of death for 20 age groups in 1990 and 2010: a systematic analysis for the Global Burden of Disease Study 2010. Lancet. 2012;380(9859):2095-128.

2. Buist AS, McBurnie MA, Vollmer WM, Gillespie S, Burney $\mathrm{P}$, Mannino DM, et al. International variation in the prevalence of COPD (the BOLD Study): a population-based prevalence study. Lancet. 2007;370(9589):741-50.

3. Mathers CD, Loncar D. Projections of global mortality and burden of disease from 2002 to 2030. PLoS medicine. 2006;3(11):e442.

4. Ito $\mathrm{K}$, Barnes PJ. COPD as a disease of accelerated lung aging. Chest. 2009;135(1):173-80.

5. Tsuji T, Aoshiba K, Nagai A. Alveolar cell senescence in patients with pulmonary emphysema. American journal of respiratory and critical care medicine. 2006;174(8):886-93.

6. Van Durme YM, Verhamme KM, Stijnen T, van Rooij FJ, Van Pottelberge GR, Hofman A, et al. Prevalence, incidence, and lifetime risk for the development of COPD in the elderly: the Rotterdam study. Chest. 2009;135(2):368-77.

7. Lamprecht B, McBurnie MA, Vollmer WM, Gudmundsson G, Welte T, Nizankowska-Mogilnicka E, et al. COPD in never smokers: results from the population-based burden of obstructive lung disease study. Chest. 2011;139(4):752-63.
8. Sabit R, Bolton CE, Edwards PH, Pettit RJ, Evans WD, McEniery CM, et al. Arterial stiffness and osteoporosis in chronic obstructive pulmonary disease. American journal of respiratory and critical care medicine. 2007;175(12):1259-65.

9. Vogelmeier C, Bals R. Chronic obstructive pulmonary disease and premature aging. American journal of respiratory and critical care medicine. 2007;175(12):1217-8. .

10. Janssens JP, Pache JC, Nicod LP. Physiological changes in respiratory function associated with ageing. The European respiratory journal : official journal of the European Society for Clinical Respiratory Physiology. 1999;13(1):197-205.

11. Blackburn EH, Gall JG. A tandemly repeated sequence at the termini of the extrachromosomal ribosomal RNA genes in Tetrahymena. J Mol Biol. 1978;120(1):33-53.

12. Aubert G, Lansdorp PM. Telomeres and aging. Physiological reviews. 2008;88(2):557-79.

13. Greider CW, Blackburn EH. A telomeric sequence in the RNA of Tetrahymena telomerase required for telomere repeat synthesis. Nature. 1989;337(6205):331-7.

14. Blasco MA. Mammalian telomeres and telomerase: why they matter for cancer and aging. European journal of cell biology. 2003;82(9):441-6.

15. Conomos D, Pickett HA, Reddel RR. Alternative lengthening of telomeres: remodeling the telomere architecture. Frontiers in oncology. 2013;3:27.

16. Okuda K, Bardeguez A, Gardner JP, Rodriguez P, Ganesh $\mathrm{V}$, Kimura M, et al. Telomere length in the newborn. Pediatric research. 2002;52(3):377-81.

17. Takubo K, Izumiyama-Shimomura N, Honma N, Sawabe M, Arai T, Kato M, et al. Telomere lengths are characteristic in each human individual. Experimental gerontology. 2002;37(4):523-31.

18. Njajou OT, Cawthon RM, Damcott CM, Wu SH, Ott S, Garant MJ, et al. Telomere length is paternally inherited and is associated with parental lifespan. Proceedings of the $\mathrm{Na}$ tional Academy of Sciences of the United States of America. 2007;104(29):12135-9.

19. Vasa-Nicotera M, Brouilette S, Mangino M, Thompson JR, Braund P, Clemitson JR, et al. Mapping of a major locus that determines telomere length in humans. American journal of human genetics. 2005;76(1):147-51.

20. Huda N, Tanaka H, Herbert BS, Reed T, Gilley D. Shared environmental factors associated with telomere length maintenance in elderly male twins. Aging cell. 2007;6(5):709-13.

21. Morla M, Busquets X, Pons J, Sauleda J, MacNee W, Agusti AG. Telomere shortening in smokers with and without COPD. The European respiratory journal : official journal of the European Society for Clinical Respiratory Physiology. 2006;27(3):525-8.

22. Von Zglinicki T. Oxidative stress shortens telomeres. Trends in biochemical sciences. 2002;27(7):339-44.

23. Steenken S, Jovanovic, S.V. How easily oxidizable is DNA? 
One-electron reduction potentials of adenosine and guanosine radicals in aqueous solution. Journal of the american chemical society. 1997;119:617-8.

24. Saito I, Takayama, M., Sugiyama, H., Nakatani, K., Tsuchida, A.,, and Yamamoto M. Photoinduced DNA cleavage via electron transfer: Demonstration that guanine residues located 5 ' to guanine are the most electron-donating sites. . Journal of the american chemical society. 1995;117:6406-7.

25. Sugiyama H, and Saito, I. Theoretical studies of GG-specific photocleavage of DNA via electron transfer: significant lowering of ionization potential and $5 \phi$-localization of HOMO of stacked GG bases in B-form DNA. Journal of the american chemical society. 1996;118:7063-8.

26. Benetos A, Okuda K, Lajemi M, Kimura M, Thomas F, Skurnick J, et al. Telomere length as an indicator of biological aging: the gender effect and relation with pulse pressure and pulse wave velocity. Hypertension. 2001;37(2 Part 2):381-5.

27. Bischoff C, Graakjaer J, Petersen HC, Jeune B, Bohr VA, Koelvraa S, et al. Telomere length among the elderly and oldest-old. Twin research and human genetics : the official journal of the International Society for Twin Studies. 2005;8(5):42532.

28. Misiti S, Nanni S, Fontemaggi G, Cong YS, Wen J, Hirte HW, et al. Induction of hTERT expression and telomerase activity by estrogens in human ovary epithelium cells. Molecular and cellular biology. 2000;20(11):3764-71 .

29. Lee J, Sandford A, Man P, Sin DD. Is the aging process accelerated in chronic obstructive pulmonary disease? Current opinion in pulmonary medicine. 2011;17(2):90-7.

30. Saldanha SN, Andrews LG, Tollefsbol TO. Assessment of telomere length and factors that contribute to its stability. European journal of biochemistry / FEBS. 2003;270(3):389-403.

31. Cawthon RM. Telomere measurement by quantitative PCR. Nucleic Acids Research. 2002;30(10):e47.

32. O'Callaghan N, Baack, N., Sharif, R., Fenech,M. A qPCR-based assay to quantify oxidized guanine and other FPGsensitive base lesions within telomeric DNA. Biotechniques. 2011;51(6):10.

33. Amsellem V, Gary-Bobo G, Marcos E, Maitre B, Chaar $\mathrm{V}$, Validire $\mathrm{P}$, et al. Telomere dysfunction causes sustained inflammation in chronic obstructive pulmonary disease. American journal of respiratory and critical care medicine. 2011;184(12):1358-66.

34. Lee J, Sandford AJ, Connett JE, Yan J, Mui T, Li Y, et al. The relationship between telomere length and mortality in chronic obstructive pulmonary disease (COPD). PloS one. 2012;7(4):e35567.

35. Rode L, Bojesen SE, Weischer M, Vestbo J, Nordestgaard BG. Short telomere length, lung function and chronic obstructive pulmonary disease in 46396 individuals. Thorax. 2012. 36. Savale L, Chaouat A, Bastuji-Garin S, Marcos E, Boyer L, Maitre B, et al. Shortened telomeres in circulating leuko- cytes of patients with chronic obstructive pulmonary disease. American journal of respiratory and critical care medicine. 2009;179(7):566-71.

37. Houben JM, Mercken EM, Ketelslegers HB, Bast A, Wouters EF, Hageman GJ, et al. Telomere shortening in chronic obstructive pulmonary disease. Respiratory medicine. 2009;103(2):230-6.

38. Mui TS, Man JM, McElhaney JE, Sandford AJ, Coxson HO, Birmingham CL, et al. Telomere length and chronic obstructive pulmonary disease: evidence of accelerated aging. Journal of the American Geriatrics Society. 2009;57(12):23724.

39. Alder JK, Guo N, Kembou F, Parry EM, Anderson CJ, Gorgy AI, et al. Telomere length is a determinant of emphysema susceptibility. American journal of respiratory and critical care medicine. 2011;184(8):904-12.

40. Lee J, Reddy R, Barsky L, Scholes J, Chen H, Shi W, et al. Lung alveolar integrity is compromised by telomere shortening in telomerase-null mice. American journal of physiology Lung cellular and molecular physiology. 2009;296(1):L57-70. 41. Faner R, Rojas M, Macnee W, Agusti A. Abnormal lung aging in chronic obstructive pulmonary disease and idiopathic pulmonary fibrosis. American journal of respiratory and critical care medicine. 2012;186(4):306-13.

42. Fauce SR, Jamieson BD, Chin AC, Mitsuyasu RT, Parish ST, Ng HL, et al. Telomerase-based pharmacologic enhancement of antiviral function of human CD8+ T lymphocytes. J Immunol. 2008;181(10):7400-6.

43. Valenzuela HF, Fuller T., Edwards, J., Finger, D., Molgora, B. Cycloastragenol extends $\mathrm{T}$ cell proliferation by increasing telomerase activity. The Journal of Immunology. 2009;182(90): 1 .

44. Pearce VP, Sherrell J, Lou Z, Kopelovich L, Wright WE, Shay JW. Immortalization of epithelial progenitor cells mediated by resveratrol. Oncogene. 2008;27(17):2365-74.

45. Sprouse AA, Steding CE, Herbert BS. Pharmaceutical regulation of telomerase and its clinical potential. Journal of cellular and molecular medicine. 2012;16(1):1-7.

46. Xia L, Wang XX, Hu XS, Guo XG, Shang YP, Chen HJ, et al. Resveratrol reduces endothelial progenitor cells senescence through augmentation of telomerase activity by Aktdependent mechanisms. British journal of pharmacology. 2008;155(3):387-94.

47. Hanahan D, Weinberg RA. The hallmarks of cancer. Cell. 2000;100(1):57-70.

48. Hanahan D, Weinberg RA. Hallmarks of cancer: the next generation. Cell. 2011;144(5):646-74. 